\title{
Co-expression network analysis revealing the key IncRNAs in diabetic foot ulcers
}

\author{
Pijun $\mathrm{Yu}^{1,2}$, Jian Guo ${ }^{2}$, Junjie $\mathrm{Li}^{2}$, Wei Chen², Tianlan Zhao ${ }^{1}$
}

\author{
${ }^{1}$ Department of Plastic and Cosmetic Surgery, The Second Affiliated Hospital \\ of Soochow University, Soochow, China \\ ${ }^{2}$ Shanghai Eighth People's Hospital, Shanghai, China
}

Submitted: 10 March 2018

Accepted: 4 July 2018

Arch Med Sci 2019; 15 (5): 1123-1132

DOI: https://doi.org/10.5114/aoms.2019.84699

Copyright @ 2019 Termedia \& Banach

\section{Abstract}

Introduction: Diabetic foot ulcers (DFUs) are the most common foot injuries leading to lower extremity amputation in diabetic patients. Recent studies showed that long non-coding RNAs (IncRNAs) played important roles in diverse biological processes. In this study, we focused on identifying differentially expressed long non-coding RNAs (IncRNAs) in DFU.

Material and methods: Real-time PCR assay was performed to validate the expression pattern of IncRNAs in DFU. Moreover, co-expression networks were also constructed to identify hub IncRNAs in DFU. Specifically, gene ontology (GO) analysis was first performed to evaluate the potential roles of differentially expressed genes (DEGs) and IncRNAs in DFU.

Results: In the present study, we identified 58 up-regulated IncRNAs and 42 down-regulated IncRNAs in DFU samples compared to non-diabetic foot skin samples by analyzing the GSE68186 dataset. Four IncRNAs (FLJ30679, LINC01193, LINC00692, and LINC00641) were observed to be up-regulated in DFU. Furthermore, we found that the down-regulated IncRNA-mediated co-expression network contained 42 IncRNAs and 700 DEGs and the up-regulated IncRNA mediated co-expression network contained 58 IncRNAs and 688 DEGs.

Conclusions: Bioinformatics analysis showed that differentially expressed IncRNAs were involved in regulating the ERK1 and ERK2 cascade, secondary alcohol biosynthetic process, centrosome duplication and DNA repair. These results suggested the potential prognostic value of IncRNAs in DFU.

Key words: long non-coding RNA, diabetic foot ulcers, co-expression network, biomarkers.

\section{Introduction}

Diabetes mellitus (DM) is a class of metabolic syndromes characterized by hyperglycemia that results from absolute or relative impairment in insulin secretion and/or insulin action, which can be induced by inherited or environmental factors [1-3]. Diabetes mellitus is one of the most common metabolic disorders in India, China, and the United States, and affected about 382 million people worldwide in 2013 [4]. Of note, an estimated 592 million DM patients will be diagnosed by 2035 [5]. Diabetic foot ulcer (DFU) is one of the most life-threatening complications of diabetes, developing in 15-25\% of diabetic patients [6]. Emerging evidence indicates that DFUs impose a substantial burden on society and family

\author{
Corresponding authors: \\ Wei Chen \\ Shanghai Eighth \\ People's Hospital \\ Shanghai 200235 \\ China \\ E-mail:dr_chenwei@163.com \\ Tianlan Zhao \\ Department of Plastic \\ and Cosmetic Surgery \\ The Second Affiliated \\ Hospital of Soochow \\ University \\ Soochow 215004 \\ China \\ E-mail: tlzhao65@163.com
}


because DFUs contribute to high morbidity, and hospitalization rates $[7,8]$. Several studies have shown DFUs to be involved in ischemia, neuropathy, impaired immune function, and infection [911]. However, there is still an urgent need to develop novel biomarkers and mechanisms for DFUs.

Non-coding RNA (ncRNA) has aroused great interest and attention in biomedical research. Long non-coding RNAs (LncRNAs) are a class of endogenous RNA molecules with length of over $200 \mathrm{nt}$ and lacking protein-coding potential [12]. Increasing reports have shown that IncRNAs play an important role in regulating genome integrity, $\mathrm{X}$ inactivation, cell cycle, cell proliferation and RNA splicing in various types of human diseases, including cancer [13-16], Hirschsprung's disease, and nonalcoholic fatty liver disease [17]. LncRNAs can regulate protein-coding genes at the epigenetic, transcriptional, and posttranscriptional levels [18]. Several studies have revealed that IncRNAs were also associated with diabetes progression. For instance, Qiu et al. found that IncRNA MEG3 was involved in diabetes-related microvascular dysfunction [19]. Recently, Sawaya et al. reported that GAS5 participated in topical mevastatin-mediated wound healing [20]. However, the functional roles of IncRNAs remained largely unclear.

In the present study, we for the first time screened differentially expressed IncRNAs in DFU by analyzing the public dataset GSE68186. The expression patterns of four IncRNAs (FLJ30679, LINC01193, LINC00692, and LINC00641) in DFU were validated using RT-PCR. Furthermore, GO and co-expression analysis were used to explore the potential roles of dysregulating IncRNAs in DFUs. These results provided useful information for exploring therapeutic candidate targets and new molecular biomarkers for DFU.

\section{Material and methods}

\section{Microarray data}

DFU gene expression data were downloaded from the study by Ramirez et al. [21], which was referenced in the Gene Expression Omnibus (GEO) database (www.ncbi.nlm.nih.gov/geo/) under accession number GSE68186. In total, 3 DFU samples and 3 non-diabetic foot skin samples were included in this dataset. We used the arrayQuality package for quality control and the limma package to apply raw data in $\mathrm{R}$ software. The normalization criteria were based on quantile normalization. mRNAs having fold changes $\geq 2$ and $p<0.05$ were selected as of significantly differential expression.

\section{LncRNA classification pipeline}

In order to identify differentially expressed IncRNAs in GSE68186, we applied a pipeline as described by Zhang [22]. The following criteria were used to identify the unique probe sets for IncRNAs from the Affymetrix array. For the probe sets with Refseq IDs, we retained those labeled as "NR_" (NR indicates non-coding RNA in the Refseq database). Next, we filtered the probe sets obtained in step 2 by filtering out pseudogenes, rRNAs, microRNAs, tRNAs, snRNAs and snoRNAs. Finally, we obtained 2448 annotated IncRNA transcripts with corresponding Affymetrix probe IDs. LncRNAs having fold changes $\geq 2$ and $p<0.05$ were selected as of significantly differential expression.

\section{Real-time reverse transcription PCR (qRT-PCR) analysis}

Total RNAs were extracted using TRIzol reagent (Sigma) following the manufacturer's instructions. Reverse transcript PCR was carried out using PrimeScript RT Master Mix (Takara, Shiga, Japan) according to the manufacturer's instructions. Real-time qPCR was performed using AceQ qPCR SYBR Green Master Mix (Vazyme Biotech co. Itd) on a Roche LightCycler 480. The PCR primers are listed in Table I. The $C t$ values were normalized using $\beta$-actin as an internal control to estimate the different expression of genes. Relative mRNA expression was calculated using the $2^{-\Delta \Delta \mathrm{ct}}$ method. Each sample was run in triplicate to ensure quantitative accuracy.

\section{PPI network construction and module selection}

The Search Tool for the Retrieval of Interacting Genes (STRING) database is an online tool designed to evaluate protein-protein interaction (PPI) information. STRING (version 9.0) covers 5214234 proteins from 1133 organisms. To eval-

Table I. Sequences of RT-PCR primers used in this study

\begin{tabular}{|lcc|}
\hline Gene symbol & Forward primer & Reverse primer \\
\hline LINC00641 & CACTTTTGCAGACCCTCACA & ACTTGACGGGTGGATTCTTG \\
\hline LINC00692 & AACTCAAACCTGCACCCATC & TTCTCTTTTGGTGCATGCTG \\
\hline FLJ30679 & ACGTGCCTGGTACATCACAA & GTTAAGTAGGCGGGCATTCA \\
\hline LINC01193 & GGTAAAGCCTCCAGTTGCCTA & GTCCAAGGAGGGTGACACTT \\
\hline
\end{tabular}


uate the interactive relationships among DEGs, we mapped the DEGs to STRING, and only experimentally validated interactions with a combined score $>0.4$ were selected as significant. Then, PPI networks were constructed using the Cytoscape software.

\section{Co-expression network construction and analysis}

The Pearson correlation coefficient of differentially expressed gene (DEG)-IncRNA pairs was calculated according to their expression value. We used the "cor" function of R software, which is common software. All parameters are default values. The co-expressed DEG-IncRNA pairs with the absolute value of the Pearson correlation coefficient $\geq 0.5$ were selected and the co-expression network was established using cytoscape software.

\section{Gene ontology (GO) analysis}

We conducted GO analysis of genes using the STRING system (https://string-db.org/). The ontology covered domains of biological processes, cellular components and molecular functions. The enriched GO terms were presented by enrichment scores. $P<0.05$ was used as the criterion for statistical significance.

\section{Statistical analysis}

All data are representative of at least three experiments of similar results performed in triplicate unless otherwise indicated. Statistical comparisons between groups of normalized data were performed using the $t$-test or Mann-Whitney $U$-test according to the test condition. A value of $p<0.05$ was considered as statistically significant with a $95 \%$ confidence level.

\section{Results}

\section{Identification and validation of differentially expressed mRNAs and IncRNAs in DFU}

In this study, we analyzed the GSE68186 dataset to identify differentially expressed genes (DEGs) in DFU. In total, 811 genes were observed to be expressed differentially, including 344 upregulated genes and 467 downregulated genes. The heat map of DEGs in DFU and non-diabetic foot skin samples is shown in Figure $1 \mathrm{~A}$.

LncRNAs have been reported to play important roles in human diseases. However, the special IncRNA expression pattern in DFU had not been revealed until now. In the present study, we identified 58 up-regulated IncRNAs and 42 down-regulated IncRNAs in DFU samples compared to non-diabetic foot skin samples by applying the IncRNA classification pipeline reported by Zhang Among these IncRNAs, AC013268.1 was the most significantly up-regulated, while AL359075.1 was the most significantly down-regulated IncRNA in DFU. The heat map of differentially expressed InCRNAs in DFU is shown in Figure $1 \mathrm{~B}$.

\section{Validation of differentially expressed IncRNAs in DFU}

To further confirm our analysis, four up-regulated IncRNAs (FLJ30679, LINC01193, LINC00692, and LINC00641) were randomly selected for further qRT-PCR validation. The expression levels of these IncRNAs from Agilent microarray data are shown in Figures $1 \mathrm{C}-\mathrm{F}$. Furthermore, we performed qRT-PCR analysis to observe IncRNAs expression levels in DFU and non-DFU samples. Four IncRNAs, FLJ30679, LINC01193, LINC00692, and LINC00641, were found to be up-regulated in DFU (Figures $1 \mathrm{G}-\mathrm{N}$ ). These results were consistent with our microarray data analysis.

\section{Construction of differentially expressed protein-protein interacting network in DFU}

Based on the information in the STRING database, PPI networks were constructed for DEGs between DFU and non-DFU. In the DFU up-regulated genes mediated PPI network, 113 nodes and 167 edges were included. UBC (degree $=25$ ), HRAS (degree $=14)$, and SRC (degree $=11$ ) were identified as key hub nodes (Figure 2 A). Meanwhile, 138 nodes and 222 edges were included in the DFU down-regulated genes mediated PPI network. We found that ATR (degree = 13), RAD52 (degree $=13), \operatorname{MRPL} 19($ degree $=11), \operatorname{RAD} 50($ degree $=11)$ and RPS3 (degree $=11$ ) were the key hub nodes with the highest connectivity degree (Figure 2 B).

\section{Co-expression network analysis}

In order to evaluate the potential roles of differentially expressed IncRNAs in DFU progression, we constructed up- and down-regulated IncRNAmRNA co-expressing networks. The co-expressed IncRNA-mRNA pairs with the absolute value of Pearson's correlation coefficient $\geq 0.9$ were selected for network construction. We found that the up-regulated IncRNA mediated co-expression network contained 58 IncRNAs and 688 DEGs (Figure 3) and the down-regulated IncRNA mediated co-expression network contained 42 IncRNAs and 700 DEGs (Figure 4). Here, we identified down-regulated LINC00638 (co-expressing with 83 DEGs), AL359075.1 (co-expressing with 82 DEGs), AC013460.1 (co-expressing with 79 DEGs), LINC00535 (co-expressing with 78 DEGs), and up-regulated FAM170B-AS1 (co-expressing with 
A
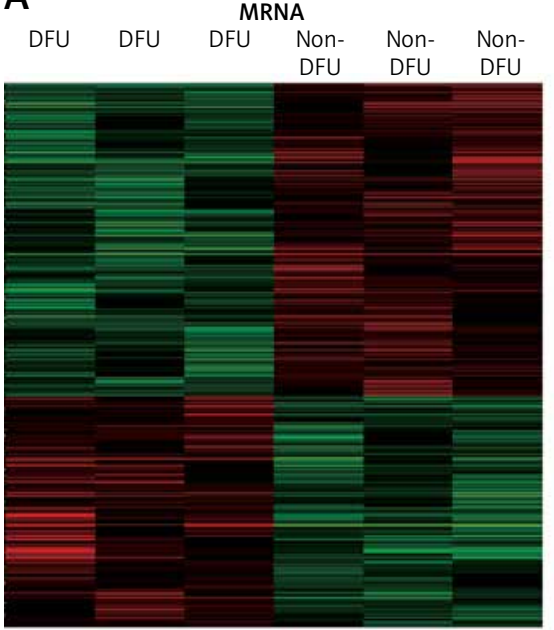

B

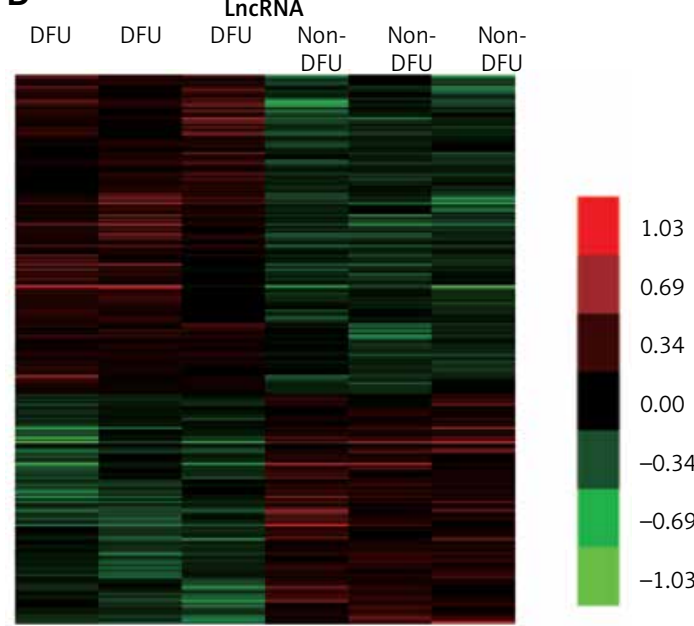

E
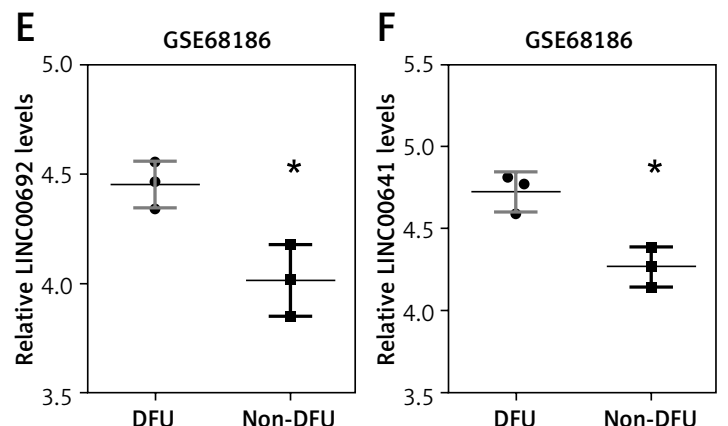

I $J$
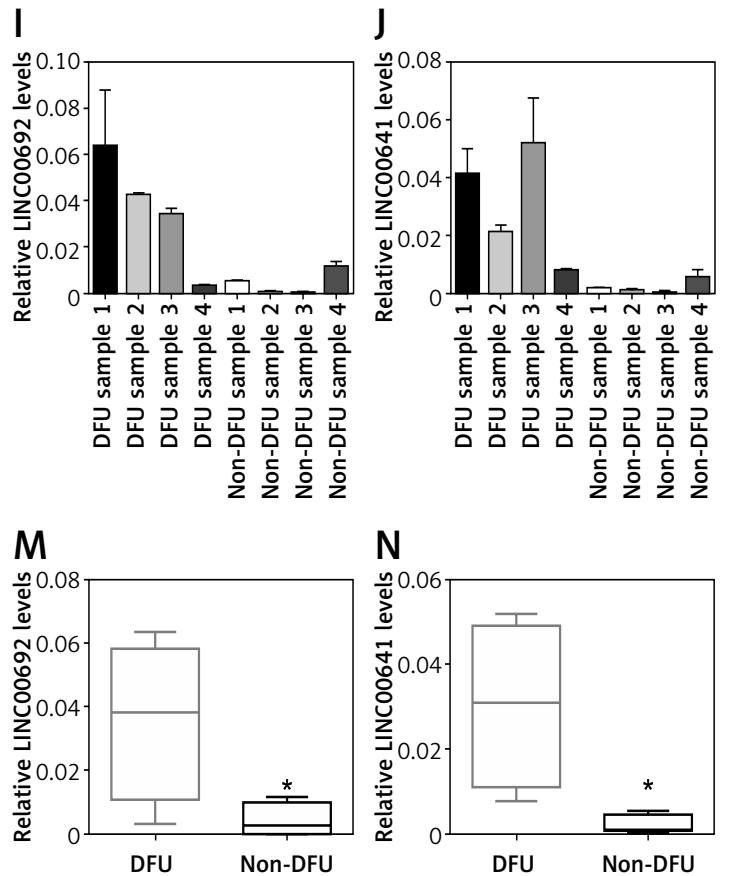

응 응 응 응 응 응 응 응

善 롬롬룜롬룜룜룜룜 言旁产言

$\mathbf{N}$

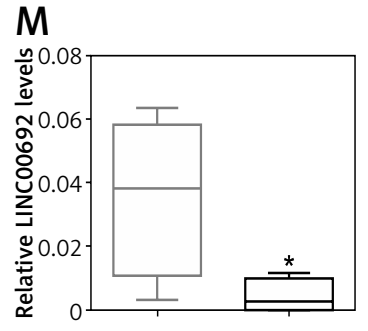

$\mathrm{K}$

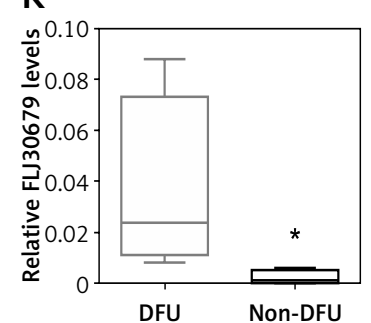

$\mathrm{L}$

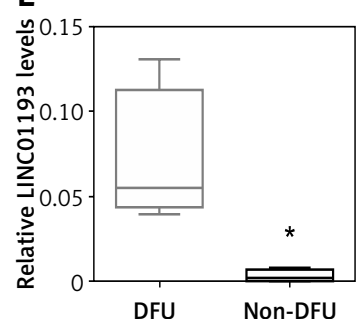

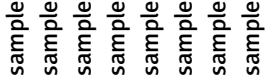
룜롬룜롬룜룜룜룜

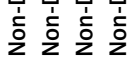

Figure 1. Identification of differentially expressed mRNAs and IncRNAs in DFU. A - Hierarchical clustering analysis shows differential gene expression in the DFU by using GSE68186. DEGs analysis shows 344 up-regulated genes and 467 down-regulated genes in DFU. B - Hierarchical clustering analysis shows differential InRNA expression in the DFU by using GSE68186. C-F - GSE68186 analysis showed that FLJ30679 (C), LINC01193 (D), LINC00692 (E), and LINC00641 (F) were up-regulated in DFU. G, K - RT-PCR analysis showed that FLJ30679 was up-regulated in DFU. H, L - RT-PCR analysis showed that LINC01193 was up-regulated in DFU. I, M - T-PCR analysis showed that LINC00692 was up-regulated in DFU. J, N - RT-PCR analysis showed that LINC00641 was up-regulated in DFU

Data are presented as the mean $\pm S D(n=3)$. Significance was defined as $p<0.05\left({ }^{*} p<0.05\right.$; ${ }^{* *} p<0.01$; $\left.{ }^{\star \star *} p<0.001\right)$; NS - not significant. 


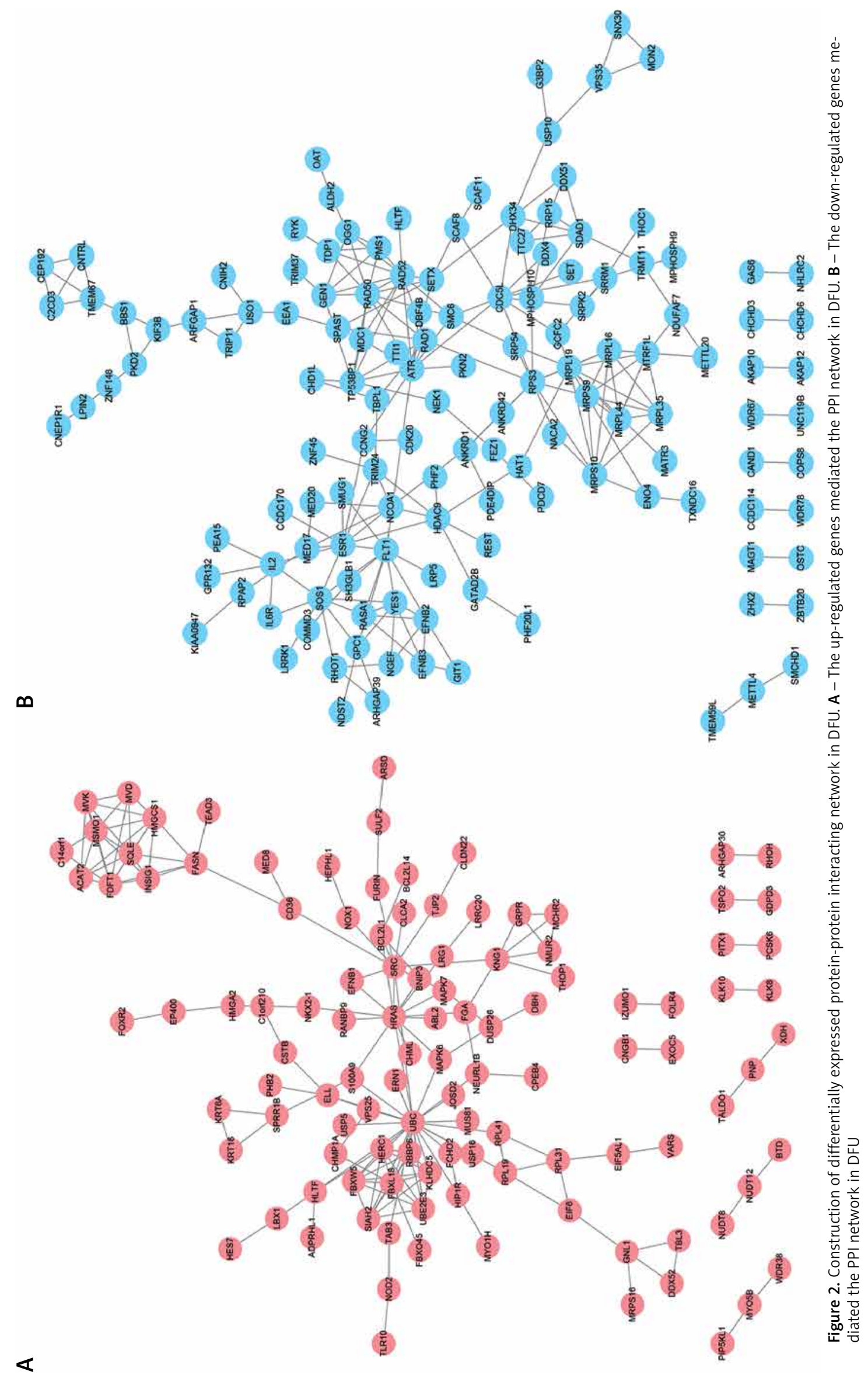




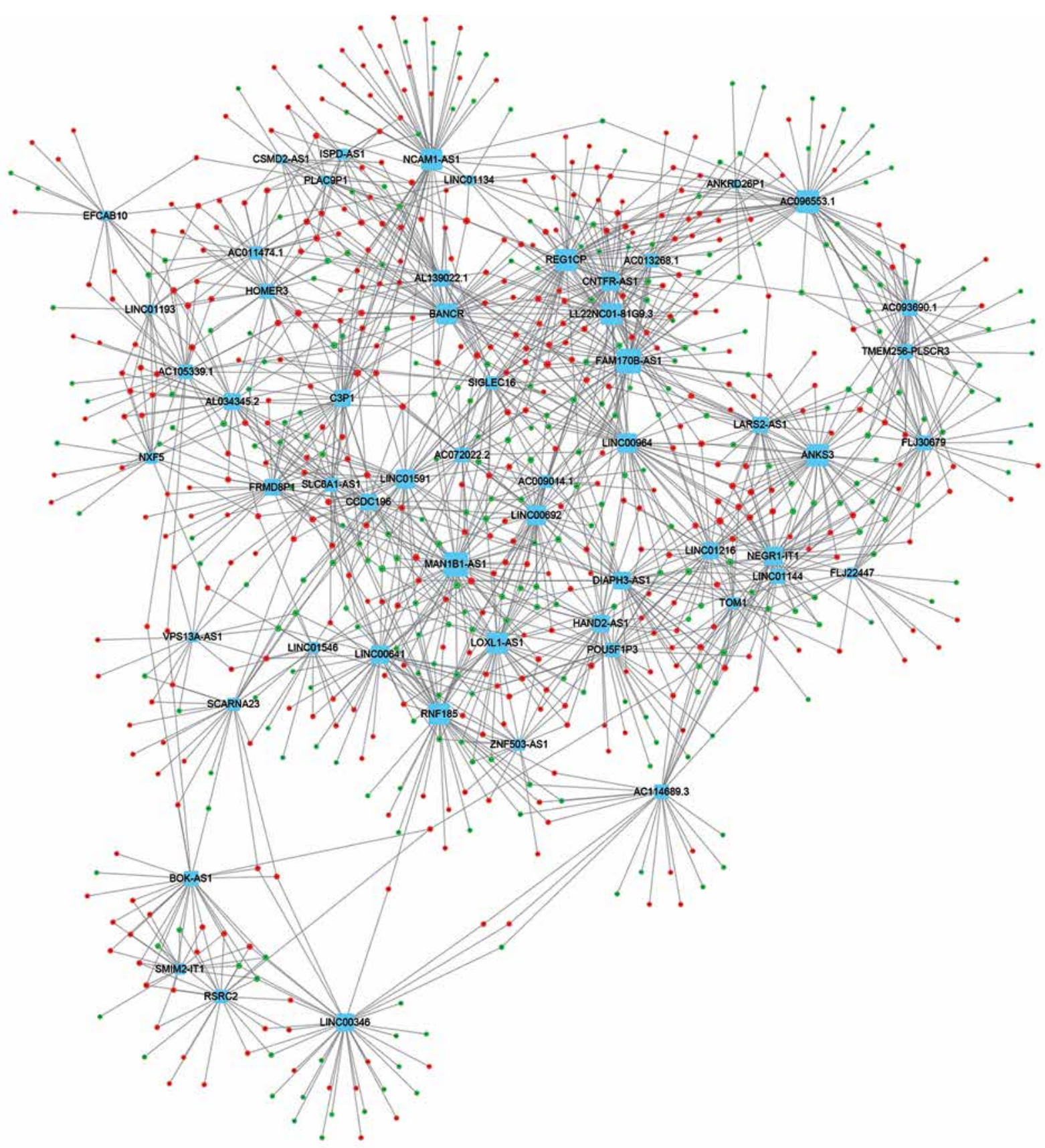

Figure 3. Co-expression network analysis of up-regulated IncRNAs in DFU. Co-expression network shows 58 IncRNAs and 688 DEGs

Blue nodes - up-regulated IncRNAs, red nodes - up-regulated mRNAs, green nodes - down-regulated mRNAs.

52 DEGs), MAN1B1-AS1 (co-expressing with 51 DEGs), AC096553.1 (co-expressing with 47 DEGs), and REG1CP (co-expressing with 45 DEGs) as key regulators in DFU.

\section{Functional annotation of differentially expressed IncRNAs}

We next performed bioinformatics analysis for differentially expressed IncRNAs in DFU by using ClueGO, which is a Cytoscape plug-in to decipher functionally grouped gene ontology and path- way annotation networks. Co-expressing DEGs of up- and down-regulated IncRNAs were classified according to the GO term. As shown in Figure 5, up-regulated IncRNAs were associated with regulation of the ERK1 and ERK2 cascade, the stress-activated protein kinase signaling cascade, the secondary alcohol biosynthetic process, neurotrophin production and cornification (Figure $5 \mathrm{~A}$ ). We also found that down-regulated IncRNAs were associated with cilium assembly and organization, centrosome duplication and DNA repair (Figure 5 B). 


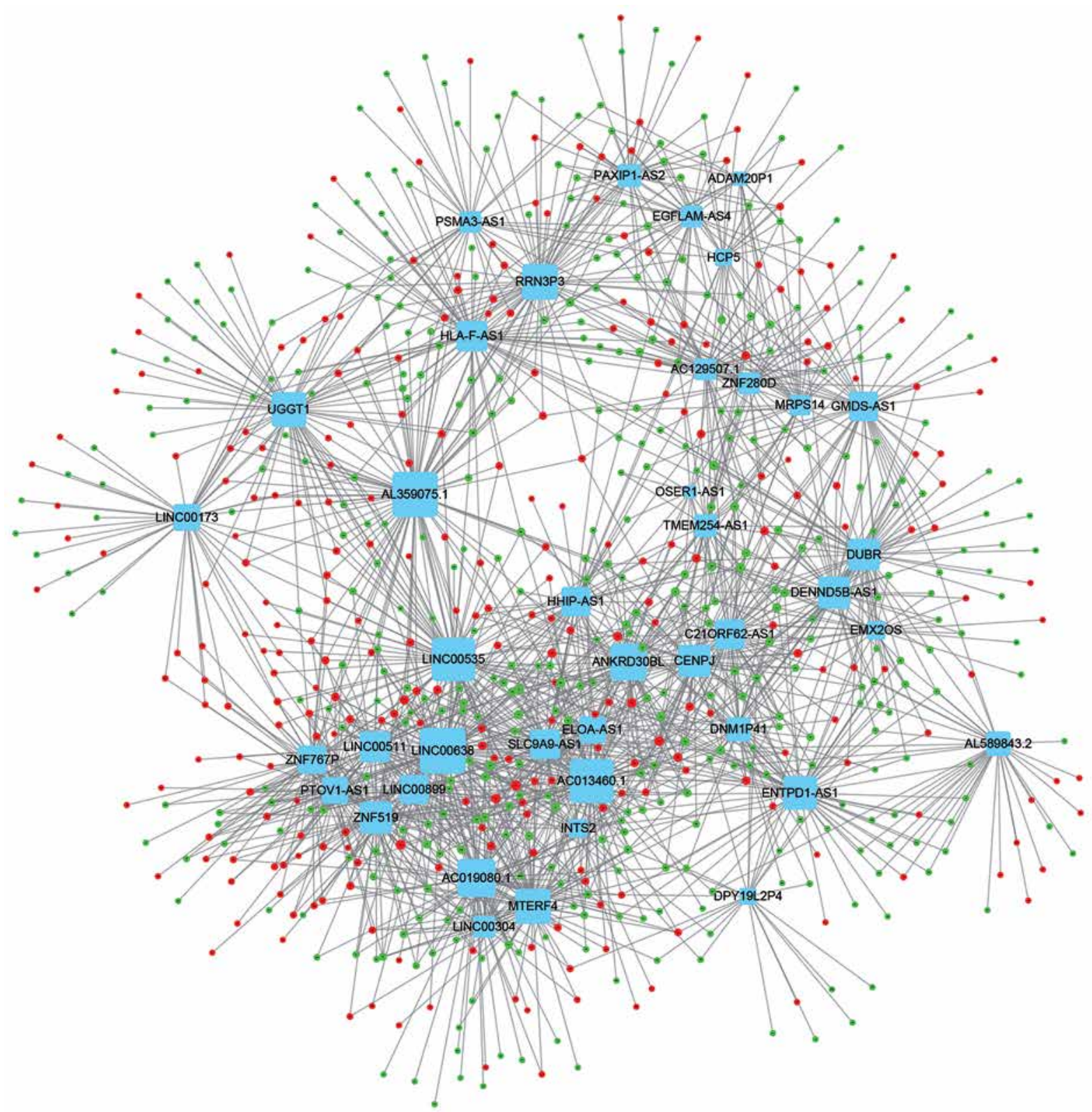

Figure 4. Co-expression network analysis of down-regulated IncRNAs in DFU. Co-expression network shows 42 IncRNAs and 700 DEGs

Blue nodes - down-regulated InCRNAs, red nodes - up-regulated $m R N A s$, green nodes - down-regulated $m R N A s$.

\section{Discussion}

Long non-coding RNAs (LncRNAs) are a class of endogenous non-coding RNA molecules with length of over 200 nt [12]. Previous studies have shown that IncRNAs play important roles in regulating a series of biological processes in human diseases, including diabetes mellitus [17]. Diabetes mellitus is one of the most common metabolic disorders. Recent reports indicated that dysregulation of IncRNAs was associated with DM progression. For example, InCRNA MALAT1 was found to be overexpressed [23] and circulating InCRNA GAS5 was down-regulated in DM compared to the normal control [24]. Furthermore, Lin et al. [25] constructed a competitive en- dogenous RNA network in type 2 diabetes mellitus which contained 351 nodes including 98 mRNAs, 86 microRNAs and 167 IncRNAs. These studies demonstrated the potential roles of IncRNAs in DM. Diabetic foot ulcer (DFU) is one of the most life-threatening complications of diabetes, developing in $15-25 \%$ of diabetic patients [11]. However, very few studies except that of Sawaya et al. have focused on exploring the special regulatory roles and expression pattern of IncRNAs in DFUs.

In the present study, we identified 58 up-regulated IncRNAs and 42 down-regulated IncRNAs in DFU samples compared to non-diabetic foot skin samples by analyzing the GSE68186 dataset. Among these IncRNAs, AC013268.1 was the 


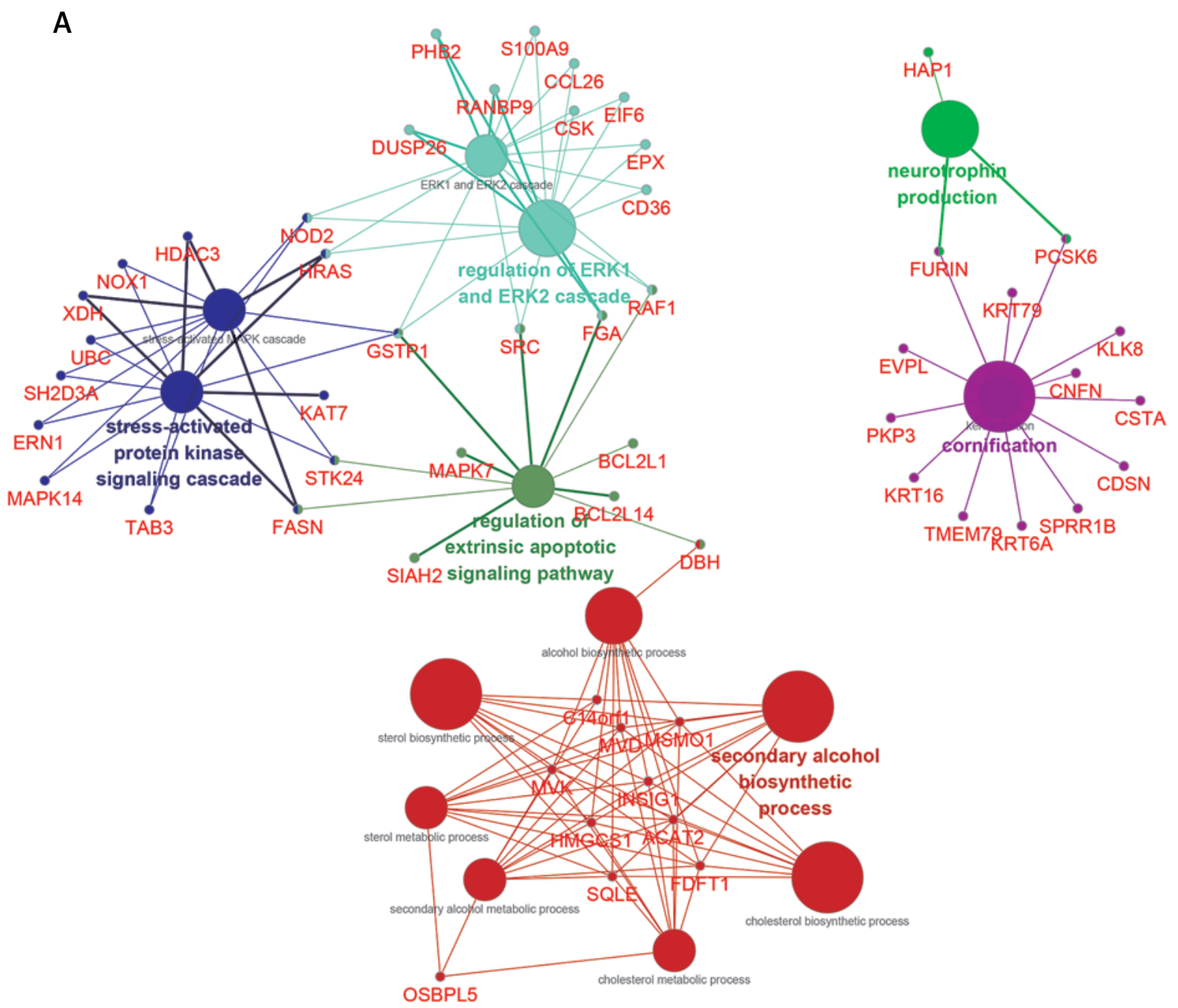

Figure 5. Functional annotation of differentially expressed IncRNAs in DFU. The GO analysis shows the up-regulated and down-regulated IncRNA associated pathways. The squares indicate IncRNAs, the arrow-headed nodes indicate mRNAs, and the circles indicate pathways

most significantly up-regulated, while AL359075.1 was the most significantly down-regulated InCRNA in DFU. To further confirm our analysis, 4 InCRNAs (FLJ30679, LINC01193, LINC00692, and LINC00641) were randomly selected for further qRT-PCR validation. Consistent with our microarray analysis, we observed that FLJ30679, LINC01193, LINC00692, and LINC00641 were up-regulated in DFU. As far as we know, this is the first study to identify differentially expressed IncRNAs globally. These results suggest the potential prognostic value of IncRNAs in DFU.

LncRNAs can regulate genome integrity, $X$ inactivation, cell cycle, cell proliferation and RNA splicing in various types of human diseases by affecting targets at the epigenetic, transcriptional, and posttranscriptional levels [26]. However, the molecular functions and mechanisms of IncRNAs in DFU have remained largely unclear. In this study, we constructed IncRNAs co-expression networks in DFU. We found that the down-regulated In-
CRNA mediated co-expression network contained 42 IncRNAs and 700 DEGs and the up-regulated InCRNA mediated co-expression network contained 58 IncRNAs and 688 DEGs. Six IncRNA (LINC00638, AL359075.1, AC013460.1, LINC00535, FAM170BAS1, and MAN1B1-AS1) were identified as key regulators in DFU progression by regulating more than 50 DEGs. Bioinformatics analysis revealed that up-regulated IncRNAs were associated with regulation of the ERK1 and ERK2 cascade, the stress-activated protein kinase signaling cascade, the secondary alcohol biosynthetic process, neurotrophin production and cornification. We also found that down-regulated IncRNAs were associated with cilium assembly and organization, centrosome duplication and DNA repair.

Of note, several proteins were also identified as key regulators in DFU. A total of 344 upregulated genes and 467 downregulated genes were found in DFU. Furthermore, PPI networks were for the first time constructed, and up-regulated 


\section{B}

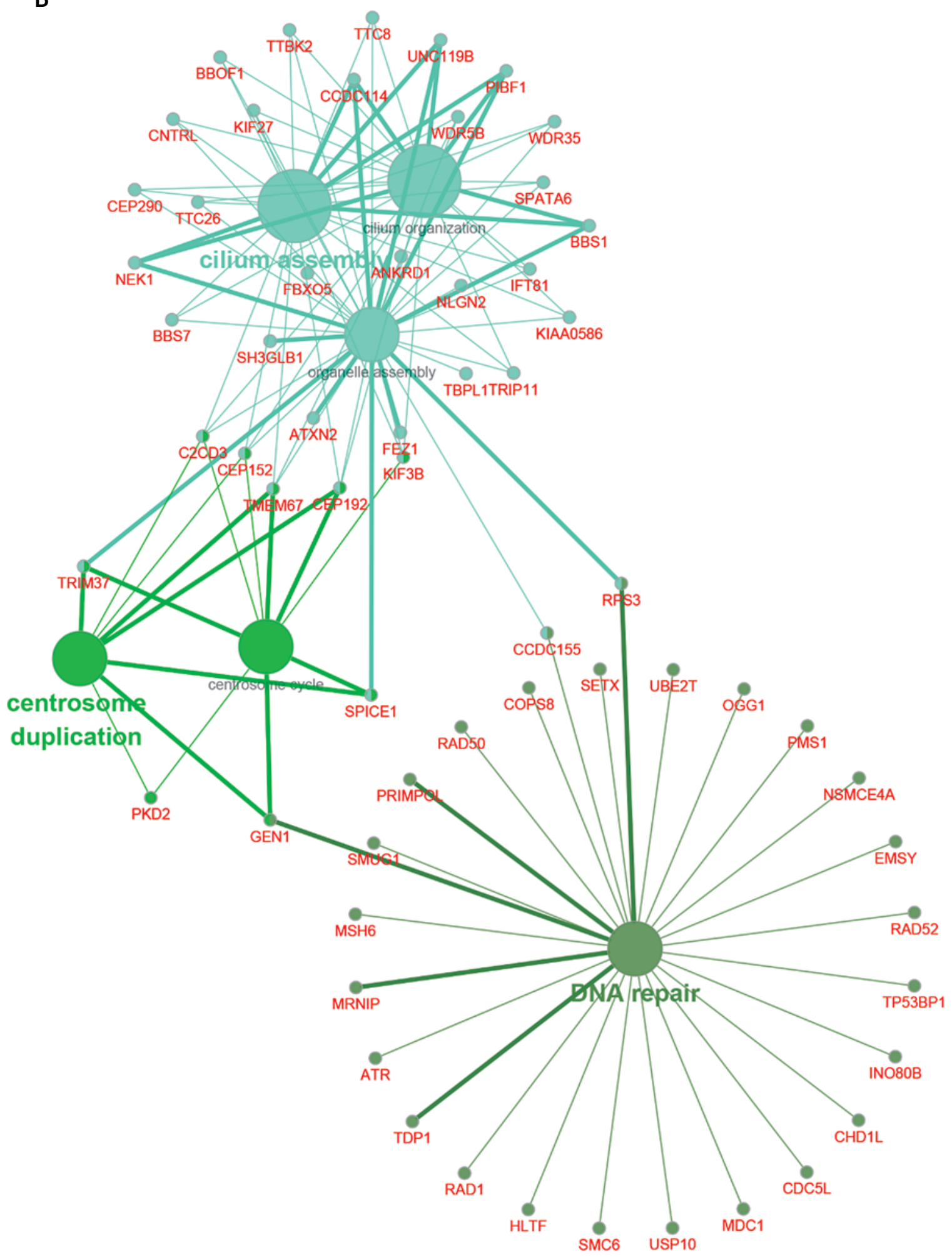

Figure 5. Cont.

UBC (degree $=25$ ) and HRAS (degree $=14$ ), and down-regulated ATR (degree $=13$ ) and RAD52 (degree $=13$ ), were identified as key proteins.

Several limitations of this study should be noted. We detected candidate IncRNAs expression levels in 3 DFU samples and 3 non-diabetic foot skin samples. The sample size is very small. In the further study, we should evaluate the correlation between expression levels of key IncRNAs and DFU progression in a large sample size, which will be 
useful for exploring whether IncRNAs could serve as DFU biomarkers. Moreover, the functional roles of key IncRNAs and proteins in DFU still need to be further validated. Loss/gain of function assays should be performed in the further study.

In conclusion, we identified 811 mRNAs and 100 IncRNAs that were significantly differentially expressed in DFU. To reveal the novel mechanisms underlying DFU progression, we for the first time constructed differentially expressed mRNA mediated PPI networks and IncRNA co-expression networks. Although several limitations should be noted in this study, these results suggest that specific IncRNAs could be valuable for exploring therapeutic candidate targets and new molecular biomarkers for DFU.

\section{Acknowledgments}

This research was also supported by the Shanghai Municipal Commission of Health and Family Planning under Grant 20154Y0198, P.R. China, and by the Jiangsu University Medical Science and Technology Development Fund Project under Grant JLY20160077, P.R. China.

\section{Conflict of interest}

The authors declare no conflict of interest.

\section{References}

1. American Diabetes A. Diagnosis and classification of diabetes mellitus. Diabetes Care 2004; 27 Suppl 1: S5-10.

2. Nathan DM, Buse JB, Davidson MB, et al. Medical management of hyperglycemia in type 2 diabetes: a consensus algorithm for the initiation and adjustment of therapy a consensus statement of the American Diabetes Association and the European Association for the Study of Diabetes. Diabetes Care 2009; 32: 193-203.

3. Ling C; Groop L. Epigenetics: a molecular link between environmental factors and type 2 diabetes. Diabetes 2009; 58: 2718-25.

4. Zimmet PZ, Magliano DJ, Herman WH, Shaw JE. Diabetes: a $21^{\text {st }}$ century challenge. Lancet Diabetes Endocrinol 2014; 2: 56-64.

5. Kharroubi AT, Darwish HM. Diabetes mellitus: the epidemic of the century. World J Diabetes 2015; 6: 850-67.

6. Lipsky BA, Berendt AR, Cornia PB, et al. 2012 Infectious Diseases Society of America Clinical Practice Guideline for the Diagnosis and Treatment of Diabetic Foot Infections A. Clin Infect Dis 2012; 54: e132-73.

7. Crews RT, Schneider KL, Yalla SV, Reeves ND, Vileikyte L. Physiological and psychological challenges of increasing physical activity and exercise in patients at risk of diabetic foot ulcers: a critical review. Diabetes Metabol Res Rev 2016; 32: 791-804.

8. Reiber G, Lipsky B, Gibbons G. The burden of diabetic foot ulcers. Am J Surg 1998; 176: 5S-10S.

9. Frykberg RG. Diabetic foot ulcers: pathogenesis and management. Am Fam Phys 2002; 66: 1655-62.

10. Boulton AJ, Kirsner RS, Vileikyte L. Neuropathic diabetic foot ulcers. N Engl J Med 2004; 351: 48-55.
11. Pozzilli P, Leslie R. Infections and diabetes: mechanisms and prospects for prevention. Diab Med 1994; 11: 935-41.

12. Moran VA, Perera RJ, Khalil AM. Emerging functional and mechanistic paradigms of mammalian long non-coding RNAs. Nucleic Acids Res 2012; 40: 6391-400.

13. Wan X, Huang W, Yang S, et al. Identification of androgen-responsive IncRNAs as diagnostic and prognostic markers for prostate cancer. Oncotarget 2016; 7 : 60503-18.

14. Feng $W$, Li L, Xu X, Jiao Y, Du W. Up-regulation of the long non-coding RNA RMRP contributes to glioma progression and promotes glioma cell proliferation and invasion. Arch Med Sci 2017; 13: 1315-21.

15. Zheng P, Li H, XU P, et al. High InCRNA HULC expression is associated with poor prognosis and promotes tumor progression by regulating epithelial-mesenchymal transition in prostate cancer. Arch Med Sci 2018; 14: 679-86.

16. Qian C, Guan M, Si C, Shen H, Jin T, Zhang T. Identification of differentially expressed profiles of IncRNAs and mRNAs in ER-negative and HER-2 positive breast cancer. Arch Med Sci Civil Dis 2017; 2: 148-60.

17. Mercer TR, Dinger ME, Mattick JS. Long non-coding RNAs: insights into functions. Nat Rev Genet 2009; 10: 155-9.

18. Kornienko AE, Guenzl PM, Barlow DP, Pauler FM. Gene regulation by the act of long non-coding RNA transcription. BMC Biol 2013; 11: 59.

19. Qiu GZ, Tian W, Fu HT, Li CP, Liu B. Long noncoding RNA MEG3 is involved in diabetes mellitus-related microvascular dysfunction. Biochem Biophys Res Commun 2016; 471: 135-41.

20. Sawaya AP, Pastar I, Stojadinovic O, et al. Topical mevastatin promotes wound healing by inhibiting the transcription factor c-Myc via the glucocorticoid receptor and the long noncoding RNA Gas5. J Biol Chem DOI: 2017jbc. M117. 811240.

21. Ramirez HA, Liang L, Pastar I, et al. Comparative genomic, microRNA, and tissue analyses reveal subtle differences between non-diabetic and diabetic foot skin. PLoS One 2015; 10: e0137133.

22. Zhang J. Designing a cost-effective and reliable pipeline leak-detection system. Pipes Pipelines Int 1997; 42: 20-6.

23. Liu J, Yao J, Li X, et al. Pathogenic role of InCRNA-MALAT1 in endothelial cell dysfunction in diabetes mellitus. Cell Death Disease 2014; 5: e1506.

24. Carter G, Miladinovic B, Patel AA, Deland L, Mastorides S, Patel NA. Circulating long noncoding RNA GAS5 levels are correlated to prevalence of type 2 diabetes mellitus. BBA Clin 2015; 4: 102-7.

25. Lin Z, Li X, Zhan X, et al. Construction of competitive endogenous RNA network reveals regulatory role of long non-coding RNAs in type 2 diabetes mellitus. J Cell Mol Med 2017; 21: 3204-13.

26. Esteller M. Non-coding RNAs in human disease. Nat Rev Genet 2011; 12: 861-74. 